\title{
A Wearable Hybrid IEEE 802.15.4-2011 Ultra- Wideband/Inertial Sensor Platform for Ambulatory Tracking
}

\author{
Michael Walsh, Salvatore Tedesco, Tingcong Ye, Brendan O'Flynn \\ Tyndall National Institute, University College Cork, \\ michael.walsh@tyndall.ie, salvatore.tedesco@tyndall.ie, tingcong.ye@tyndall.ie, brendan.oflynn@tyndall.ie
}

\begin{abstract}
Ultra-Wideband (UWB) transceivers and low-cost micro electro mechanical systems (MEMS) based inertial sensors are proving a promising hybrid combination for location specific wearable applications. While several hybrid systems have been proposed to date, current approaches consider inertial sensors and UWB as ad-hoc components working in isolation. As a result issues surrounding extensive infrastructure requirements, synchronization, and limitations associated with the mutual sharing of inertial data have arisen. In an attempt to address such limitations, this paper presents a fully-coupled architecture whereby standardised IEEE 802.15.4-2011 UWB is employed for both ranging and as a mechanism for exchanging inertial data between the nodes of a network. A proof-of-concept system is implemented and tested for a single ambulatory use case scenario. Basic fusion algorithms are employed and the preliminary results show the benefits of a fully-coupled approach when compared with traditional standalone inertial navigation.
\end{abstract}

\section{Keywords}

IEEE 802.15.4-2011 UWB, Inertial Sensors, MEMS, Fully Coupled Architecture, Hybrid System, Wearable.

\section{INTRODUCTION}

Location-based services are becoming a vital part of people's lives and are integral in many wearable applications including navigation, tracking, digital sports and healthcare provision [1]. Such services are based on localization techniques whose aim is the timely determination of the positional state of a moving object. The overall performance, reliability, cost and complexity of the localization are strongly influenced by the adopted technology. In an outdoor environment, global navigation satellite systems (GNSS) represent a solution and have been used in a wide range of applications, such as tracking, asset management, transport navigation and geodetic surveying [2]. However, such a system unaided is ineffective for indoor positioning and, to address this shortcoming, alternative technologies have been developed, which include infrared radiation (IR), ultrasound, and Radio Frequency (RF) technologies such as Wi-Fi, Radio Frequency Identification (RFID), Bluetooth, Zigbee and Ultra-Wideband (UWB).

Permission to make digital or hard copies of all or part of this work for personal or classroom use is granted without fee provided that copies are not made or distributed for profit or commercial advantage and that copies bear this notice and the full citation on the first page. To copy otherwise, to republish, to post on servers or to redistribute to lists, requires prior specific permission and/or a fee.

UWBAN 2014, September 29-October 01, London, Great Britain Copyright (C) 2014 ICST 978-1-63190-047-1

DOI 10.4108/icst.bodynets.2014.258233
IR and ultrasound approaches can suffer from several drawbacks including form factor limitations, expensive hardware and maintenance cost, line of sight constraints and interference [3-4]. RF technologies offer a degree of flexibility for indoor positioning particularly in the wearable context in terms of cost, form factor and ability to operate in non-line-of-sight (NLOS) conditions. Among the numerous RF positioning techniques proposed, the most commonly utilized are based on determining the angle, Received Signal Strength (RSS) and/or time-of-arrival of the received wireless signal.

In general, time-based algorithms present more robustness and an enhanced mitigation of the multipath effects when compared to angle/RSS-based techniques [4]. While, theoretically, every RF technology could realize positioning employing a time-based approach, the overall performance varies according to the signal bandwidth and the signal-to-noise ratio (SNR) in line-of-sight (LOS) areas. UWB technology, with its unique characteristic of wide signal bandwidth $(>500 \mathrm{MHz})$ and consequent high time resolution property, represents one of the most promising technologies for ranging and indoor positioning, with an accuracy in the order of decimetres [5].

Moreover, the relatively recently (2003) deregulated technology exhibits additional benefits, including ultra-low power operation, high data rates, enhanced multipath immunity when compared with other RF technologies, robustness to jamming, and low electromagnetic (EM) interference allowing UWB to coexist with other wireless devices. This, in turn, makes UWB a particularly attractive implementation for bio-medical applications, including monitoring of continuous medical signals, wireless capsule endoscope and gait analysis [6-7]. Recent works have also considered the characterization of the UWB channel model and its suitability as a wireless technology for wearable applications [8-9]. This work demonstrated that, when the UWB electric field is perpendicular to the human body, the EM field tends to propagate along the body surface, while the EM penetration inside the body is small.

However, despite the abovementioned advantages, UWB implementations are still prone to reduced performance due to multipath effects, particularly in NLOS conditions. Indeed, these effects are most prominent while tracking moving objects or persons, a key constraint for wearable applications. Therefore in order to improve the system performance in terms of accuracy, robustness, and multipath/NLOS compensation, a number of hybrid indoor positioning systems combining the complementary advantages of UWB and inertial sensors (3D accelerometer and $3 \mathrm{D}$ gyroscope) have been proposed [10-13].

The adoption of a stand-alone inertial sensor approach to navigation has long been established for various applications in avionics and robotics [14-15]. With this technology, the current position and orientation are estimated by accumulating movements determined using the on-board sensors. However, 
even though inertial data present regularity and independence from any existing infrastructure, accurate position tracking is provided only for short periods of time, being prone to drift for longer timescales.

The integration of UWB and inertial measurement units (IMUs) has therefore two primary benefits. Firstly, IMU data can be employed to compute the travelled trajectory when UWB measurements are absent due to range limitations or adverse NLOS conditions, and secondly, UWB-based positioning can supplement IMU data to discriminate between accurate measurements and data corrupted by noise and drift. To date however proposed solutions have implemented the two technologies in isolation, for example in [10-13], where the IMU navigation and UWB ranging execute separately and their respective data are fused a posteriori using particle or Kalman filter algorithms. This approach limits the achievable synchronization between the inertial sensor data and UWB ranging measurements, reducing the achievable accuracy of the system. These methodologies are also limited in that the nodes are incapable of exchanging inertial or positional data with other network nodes given the UWB channel is dedicated to ranging alone. The solution in many cases is to supplement the system with an additional wireless technology, which, in turn, increases the cost and size of the infrastructure and further complicates data synchronization.

A potential solution is to fully couple the adopted technologies, which implies that the UWB communication channel is used both for ranging and as mechanism for transmitting inertial, positional and other useful information e.g. physiological. This reciprocal data distribution allows several nodes to track one another and for each node to consider its mobile neighbours as anchors at intelligently arrived at spatiotemporal points. This approach therefore has the potential to reduce the overall number of anchors needed for localization.

In this paper, a novel fully-coupled hybrid UWB/IMU proof-ofconcept is proposed, combining the emerging IEEE 802.15.42011 UWB transceiver technology and MEMS inertial sensors. The rest of the paper is organised as follows: firstly, in Section II, the IMU orientation estimation, the UWB ranging methodology and the novel hybrid architecture are discussed. The proposed solution is then validated for one practical ambulatory scenario, and results are presented (Section III). Finally conclusions are drawn and future developments are outlined (Section IV and Section $V$, respectively).

\section{FULLY-COUPLED ARCHITECTURE: DESCRIPTION}

The hybrid system proposed in this work, which represents a node in a wireless network, consists of two modules, an IMU and an IEEE 802.15.4-2011 UWB transceiver. The following subsections deals with the description of the orientation estimation from the inertial data, the ranging technique through UWB, and the fully-coupled architecture to synchronize all these measurements for both transmitting and receiving nodes.

\subsection{Orientation Estimation}

The orientation of an inertial module can be symbolized with different mathematical representations. Although Euler angles (roll, pitch, and yaw) are the most intuitive method to describe an object's orientation, they present some singularities, which make such a representation not particularly attractive. To deal with this problem, quaternion algebra is generally used to show orientation. A first approach to extrapolate orientation from inertial data is by numerically integrating the angular rate measured by a 3D gyroscope. However, gyroscopes are primarily affected by bias, making the final orientation subject to boundless drift error. Another method considers the use of accelerometers and magnetometers to provide orientation estimation. Whilst accelerometers produce a reference in the vertical plane, magnetometers arrange the same information in the horizontal plane. Hybrid approaches to date employ accelerometers and gyroscopes alone [10-12], where a key constraint is the requirement that some acceleration must be present at all times so as to obtain heading (i.e. the angle around the vertical). Nevertheless, all such sensors are also affected by white noise, bias, and magnetic disturbances; therefore various algorithms are adopted as optimizers to minimize the mismatching error in orientation, due to the different nature of the sensors adopted for the vertical and the horizontal references.

It is worth observing that gyroscopes are reliable only in the short-term and cannot provide an absolute orientation reference. On the other hand, accelerometers are sensitive to high dynamic motions; and magnetometer outputs can be corrupted by ferromagnetic materials in the proximity of the sensor; thus, are more effective in the long-term. For these reasons, a complementary filter represents a useful solution so as to fuse the outputs from sensors with complementary spectral characteristics. In particular, the quaternion obtained from accelerometer and magnetometer passes through a low-pass filter, whilst the gyro-based quaternion through a high-pass filter. Resulting signals are finally combined to provide an accurate and robust orientation. It is for this reason that the approach adopted in this work employs accelerometer, gyroscope and magnetometer data and complete details can be found in [16].

Accuracy inherently depends also on the quality of the inertial sensors, which are affected by errors caused during large scale manufacture, assembly and packaging. Therefore, the adoption of a calibration procedure [17] capable of compensating for accelerometers bias, gyroscope bias, and hard/soft iron effects is adopted for this work. Title and Authors

\subsection{Ranging Measurements}

A number of challenges still remain when deploying UWB ranging particularly in mobile situations including nonsynchronized wireless network, signal acquisition, multi-user interference, multipath effects, and NLOS propagation.

In order to minimize the effects of these hardware and environmental issues, a Symmetric Double-Sided Two-Way Ranging (SDSTWR) algorithm has been recently proposed and developed for personal area networks [18]. It relies on the estimation of the Time-of-Flight (TOF) of an UWB signal pulse traveling back and forth between two UWB modules (known as the leader and the follower). For the sake of clarification, the leader transmits a signal to the follower, which, in turn, retransmits the signal back to the leader after a system processing delay $(D E L)$. When the leader receives the signal from the follower, it calculates its Round-Trip-Time (RTT). This is followed by another signal containing all the timing values including an additional system processing delay transmitted from the leader to the follower, which in turn estimates its own RTT. 
The distance $d$ is finally calculated employing the two RTT and the two delays.

$$
T O F=\frac{R T T_{\text {leader }}-D E L_{\text {follower }}+R T T_{\text {follower }}-D E L_{\text {leader }}}{4}
$$

$$
d=c \bullet T O F,
$$

where $c$ indicates the speed light. The multiple exchanges of timing data enable the SDSTWR algorithm to compensate for crystal frequency offsets, further minimizing the residual error. However, ranging performance could be still limited in dense cluttered environments and in NLOS situations. These phenomena are addressed in this work by employing a Bilateral Transmitter Output Power Control (BTOPC) technique [19] which when coupled with the SDSTWR algorithm is shown to stabilize the bidirectional UWB channel. Both methodologies are implemented in this paper as part of the ranging paradigm.

\subsection{Proposed Architecture}

For many applications it is a requirement that a subject's movements can be tracked both locally and remotely. "Locally" implies that the same node is able to self-locate using the measurements of the on-board IMU and UWB modules. Remote tracking indicates that the tracking is carried out by a second node (such as, a peer node or a beacon) to which the first node is transmitting all the measured data. In order to implement efficient mutual localization; a "local" and a "remote" synchronization mechanism must be realized.

Considering the IMU components of two independent hybrid modules, it is not possible to assume a synchronous or constant sampling rate, due to elements such as clock drift and asynchronous event triggering. The UWB ranging sampling rate generates data with an irregular cadence also. This is due to the dependence, in the ranging estimation, on TOF measurements and processing delays, which, in turn, are strongly correlated with hardware limitations and the varying distance associated with the ambulatory environment.

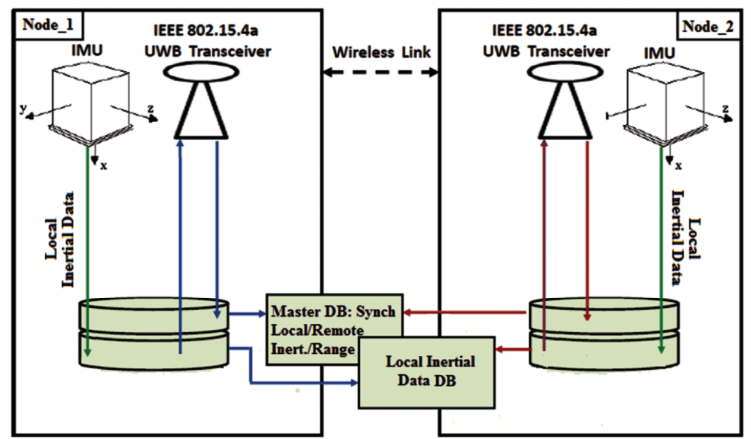

Figure 1. Fully-coupled 802.15.4-2011 UWB\IMU architecture including ranging, wireless communications and local and remote data synchronization

A fully-coupled architecture is proposed in this paper in order to minimize such a synchronization issue and to make remote tracking possible. Figure 1 illustrates the architecture schematic for a 2 node scenario. Data communications is shown in Figure 2 where the Node 1 IMU module estimates its 3D orientation and stores it chronologically in a Local Inertial database, which is periodically updated. Simultaneously, when the on-board UWB transceiver is transmitting a wireless signal to perform ranging, the latest inertial data accumulated in this database is integrated into the transmitted UWB packet. Figure 2 highlights that the temporal difference between the UWB signal and the inertial orientation is quantifiable and is equal to the IMU 3D orientation algorithm sampling time (for instance $1 \mathrm{~ms}$ in case of a $1 \mathrm{kHz}$ sampling rate). Subsequently, the UWB transceiver in Node 2 receives the packet and, in accordance with the SDSTWR algorithm, re-transmits a signal to Node 1, contained in which is the latest inertial data, stored in Node 2's Local Inertial database, along with the distance estimated from the received ranging information. Upon receiving this transmission, Node 1 is subsequently able to complete the SDSTWR algorithm distance calculation "locally" and to associate to this value the latest locally generated inertial sample. In addition it receives the distance estimated "remotely" by Node 2, together with the associated inertial sample.

Again from Figure 2 the temporal difference between the distance measurements for the two nodes is quantifiable and is equal to the sum of a TOF and a system processing delay. The lag between the inertial samples is equal to the sum of the TOF and the IMU 3D orientation algorithm sampling time. In case of typically indoor short-range areas (up to $100 \mathrm{~m}$ ) and with high IMU orientation sampling rate $(>1 \mathrm{kHz})$, the two contributions have the same weight. Vice versa, with a low IMU sampling frequency, the TOF contribution can be neglected in comparison to the second variable. For the two nodes scenario in question all four parameters are then stored in a second Master database where they are temporally aligned. The Master database is populated by each node communicating in the network enabling local or remote determinations to be made on a continual basis with measureable and compensable temporal offset.

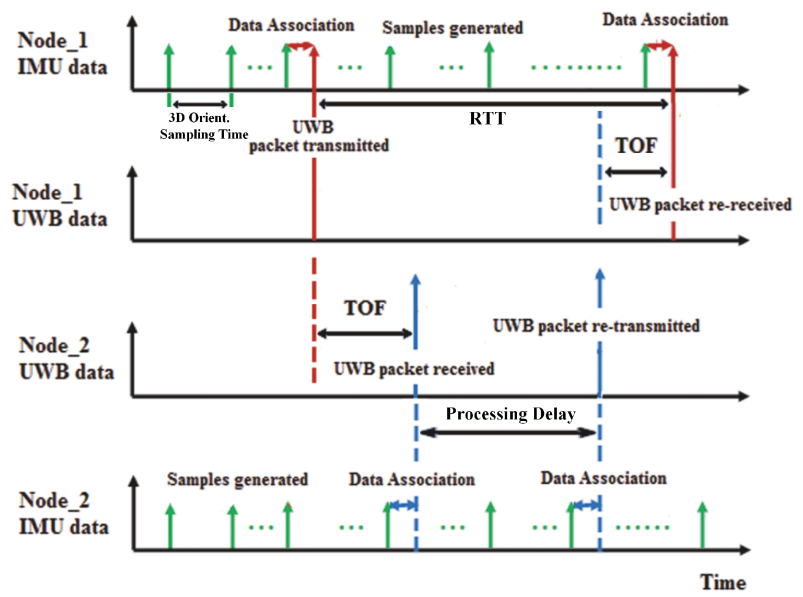

Figure 2. Fully-coupled 802.15.4-2011 UWB\IMU Data Communication

\section{RESULTS AND DISCUSSION}

This section deals with a single use case scenario implementing a practical proof of concept system for the architecture introduced in Section II. The experiment entails the use of two nodes (for the purposes of the experiment known as the mobile and beacon nodes respectively), each comprising of an IMU and a UWB module. More specifically, the IMU module (Figure 3a) is based 
on the modular Tyndall $25 \mathrm{~mm}$ mote platform [20], which consists of an array of 3D accelerometer, 3D gyroscope, and 3D magnetometer coupled with a high resolution analog-to-digital converter (ADC). The UWB prototype (Figure 3b), developed by Decawave, is the world's first IEEE 802.15.4-2011 standard compliant UWB transceiver. 3D orientation is estimated by the IMU in real-time 10 times per second employing the wellestablished low computational methodology in [16]. The 802.15.4-2011 UWB module, depending on range, calculates the distance between the two transceivers approximately every 0.6 seconds. Table I details the 802.15.4-2011 UWB signal specifications adopted for the experiment. SDSTWR [18] and BTOPC [19] algorithms are coupled to provide stable channel UWB measurements. Inertial and ranging data from remote and local nodes are synchronized from both sides by means of the fully-coupled architecture in Figure 1.

In the experimental scenario (see Figure 7), one of the nodes represents a non-mobile beacon with known position, considered $(0 ; 0)$, whilst the second node moves counter-clockwise on a $2 \mathrm{D}$ plane according to a path representing a Greek cross with equal arm width and length. The length of each segment of the cross is $2 \mathrm{~m}$ and the route topology has been chosen in order to have a set of several orientation changes (12 in total) in correspondence with varying range. The start point of the mobile path coincides with the end point and is located at $(2 ; 0)$.

Table 1. 802.15.4-2011 UWB Signal Specification

\begin{tabular}{|c|c|}
\hline Preamble Length & 1024 \\
\hline Data Rate & $850 \mathrm{kbps}$ \\
\hline Centre Frequency & $4 \mathrm{GHz}$ \\
\hline Max Transmit Power & $-13.5 \mathrm{dBm}($ FCC) \\
\hline Min Transmit Power & $-31.5 \mathrm{dBm}$ (device) \\
\hline IEEE 802.15.4-2011 Channel Index & 2 \\
\hline Pulse Repetition Frequency & $16 \mathrm{MHz}$ \\
\hline
\end{tabular}

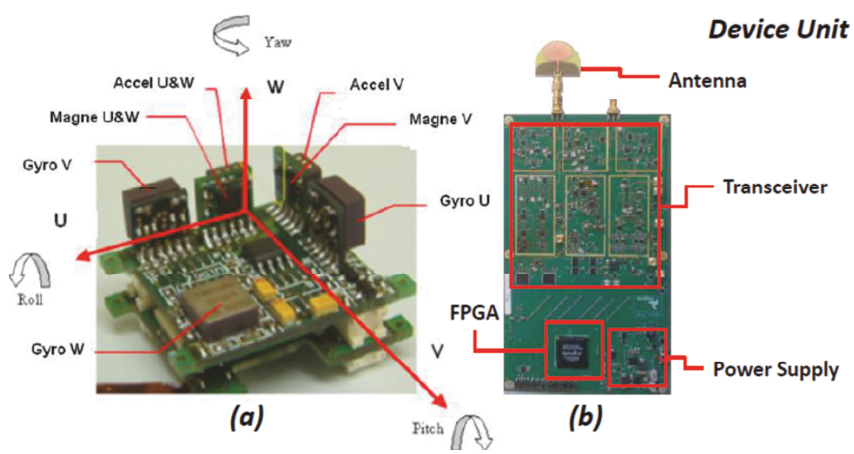

Figure 3. (a) IMU module, and (b) IEEE 802.15.4-2011 UWB transceiver prototype
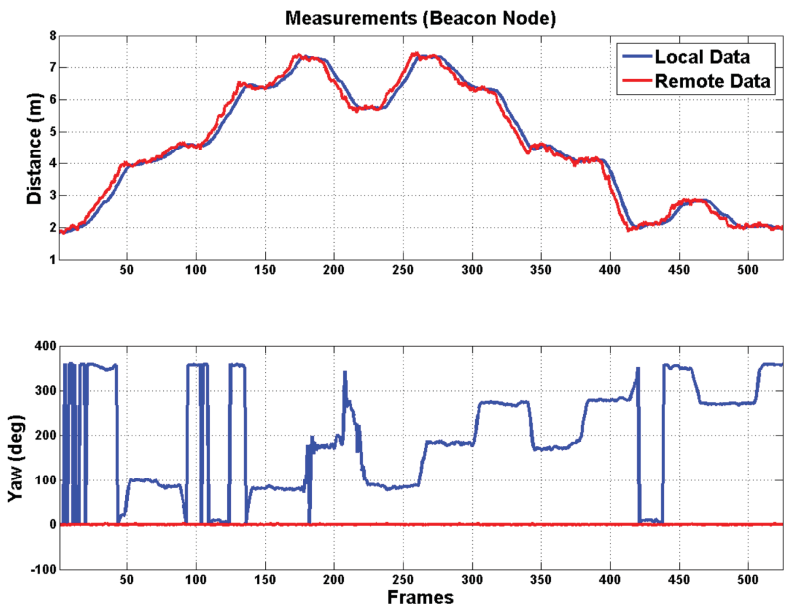

Figure 4. Local and remote orientation/range data observed at the beacon node. Note the beacon node is stationary and therefore observes no local change in orientation during the experiment.

\subsection{Synchronous Data Communication}

In Figure 4, the local and remote ranging/orientation data collected during the test by the beacon node are presented. The same information estimated by the mobile node is illustrated in Figure 5. It can be observed that, as expected, the local data at the beacon is closely related to the remote information available to the mobile object and vice versa. Such bilateral mutual and synchronous data sharing is enabled through the use of the fullycoupled architecture. The beacon node being stationary throughout observes no local change in orientation during the experiment.
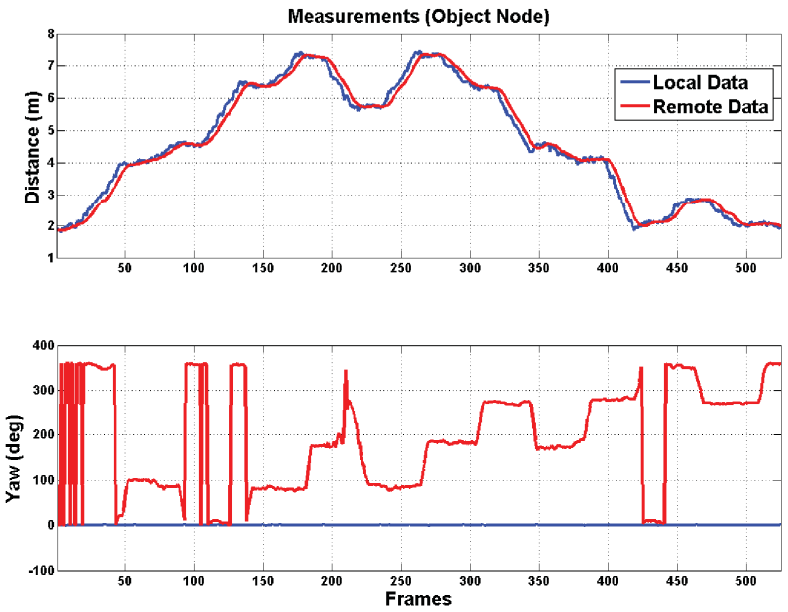

Figure 5. Local and remote orientation/range data observed at the mobile node. Note the beacon node is stationary and therefore observes no local change in orientation during the experiment. 


\subsection{D Greek Cross Navigation Experiment}

Secondly to demonstrate the applicability of the proposed architecture a 2D indoor localization algorithm is executed on the datasets presented in Figures 5 and 6. The algorithm used for comparison is basic and relies on a pure inertial navigation system (INS) approach. In tandem a temporally constant UWBbased error correction technique is applied to the output of the INS algorithm. It is assumed that the beacon represents the origin of a Cartesian coordinate system whose orientation is employed to establish a reference frame. According to the INS technique, the position of the mobile node compared to the beacon can be calculated using the following equations:

$$
\begin{aligned}
&\left\{\begin{array}{r}
X_{N+1}=X_{N} \pm v_{x, N+1} \bullet d t \\
Y_{N+1}=Y_{N} \pm v_{y, N+1} \bullet d t
\end{array}\right. \\
& \text { with }\left\{\begin{aligned}
v_{x, N+1} & =\bar{v} \bullet \cos \left(\Phi_{N+1}\right) \\
v_{y, N+1} & =\bar{v} \bullet \sin \left(\Phi_{N+1}\right)
\end{aligned}\right.
\end{aligned}
$$

where $\left(X_{N+1}, Y_{N+1}\right)$ indicates the next position of the mobile node, $\left(X_{N}, Y_{N}\right)$ is the current position, $d t$ is the sampling time, $\bar{v}$ is the magnitude of its speed, and $\Phi_{N+1}$ is the difference between the orientations estimated by the two nodes. It is worth pointing out that, in the previous formula, the sign ' + ' is necessary in case the mobile node moves forward, the opposite when it moves backward. Although the discrimination between the two movements is trivial due to the presence of a gyroscope in the IMU, for simplicity, it is assumed that the mobile node moves in the forward direction only. Furthermore, even though the speed magnitude could be estimated by means of a double integration of the acceleration (after the transformation from the body to the global reference and a gravity subtraction), it is assumed to be uniform for this experiment.

The hybrid methodology as illustrated in Figure 6 extrapolates a line drawn between the beacon node located at $(0,0)$ and the position currently estimated by the INS approach $\left(X_{N+1}, Y_{N+1}\right)$.

The corrected position of the mobile node is taken as the point of intersection $\left(X_{C O R}, Y_{C O R}\right)$ of a circle with centre $(0,0)$ and with radius equal to the current UWB ranging measurement $d$ as per the SDSTWR algorithm and (1). The point so calculated represents the new estimated position of the mobile node and is taken as $\left(X_{N}, Y_{N}\right)$ in the subsequent iteration of the INS algorithm in (2).

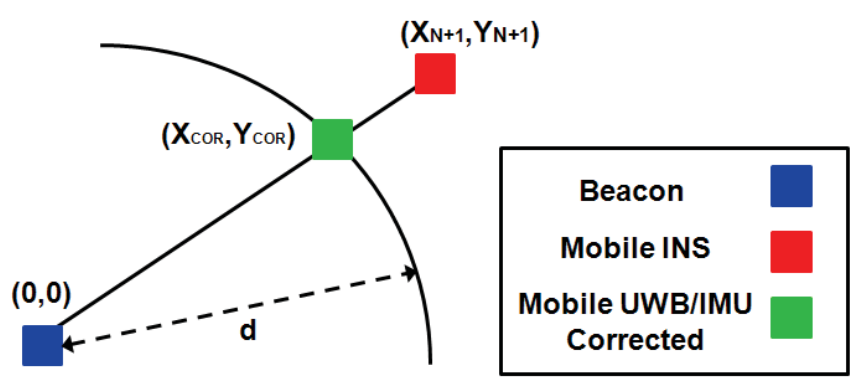

Figure 6. Hybrid UWB/IMU correction for INS drift prone algorithm.

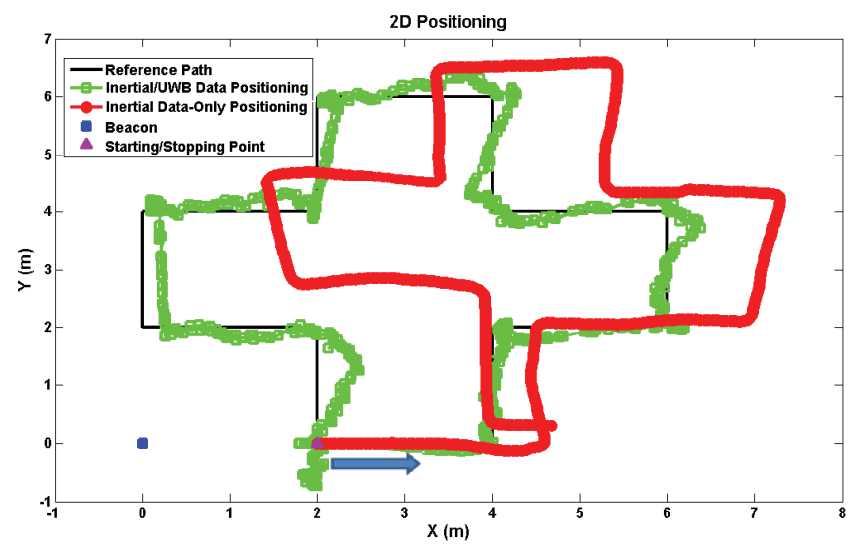

Figure 7. 2D Positioning with INS approach (red curve) and INS+UWB correction mechanism approach(green curve).

In Figure 7 , the $2 \mathrm{D}$ positioning performance of the two approaches (INS and INS+UWB) are presented. Results for the standalone INS approach (red line) highlight the methodology is prone to drift due to the noise characteristics of the inertial sensors. Results for the hybrid approach (green line) show that the correction for sensor drift provided by fully coupled UWB enhances the overall tracking performance of the system substantially.

The experimental error as represented in Figure 8 is calculated in correspondence with each turn while traversing the predefined Greek cross reference path. For the INS case we can see that the error is initially small however it continually increases throughout the experiment reaching 2.6 metres and looks toward becoming unbounded. The UWB-based correction on the other hand maintains the error almost uniformly $(<55 \mathrm{~cm})$ during each of the 12 turns of the experimental procedure. While the resulting error is not insubstantial it does however indicate the potential for the proposed architecture in the ambulatory context.

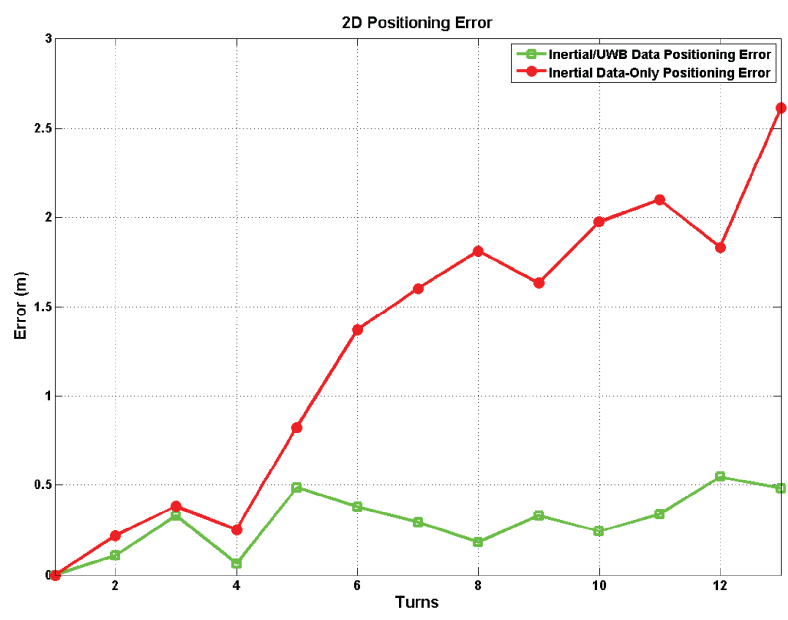

Figure 8. Error comparison between standalone INS approach (green curve) and INS+UWB correction approach (red curve) calculated at every corner of the traversed path. 


\section{CONCLUSIONS}

In this paper IEEE 802.15.4-2011 Ultra-Wideband (UWB) was suggested as a high potential technology for wearable applications both as a localisation tool and as a means of communicating sensor data. A novel hybrid fully-coupled architecture was introduced comprising of micro electro MEMS based inertial sensors and emerging IEEE 802.15.4-2011 UWB transceiver technology. The architecture was designed for synchronous remote and local tracking for multiple mobile node networks. A proof-of-concept system was fully implemented integrating the Tyndall $25 \mathrm{~mm}$ inertial measurement platform with 802.15.4-2011 compliant UWB. Results presented for a two node ambulatory use case scenario demonstrated synchronous inertial sensor and ranging data communication. A 2D Greek cross navigation experiment illustrated the improvements afforded by the fully coupled hybrid approach when compared with a traditional inertial navigation technique.

\section{FUTURE WORK}

While significant efforts have been made to design and implement hybrid UWB/IMU technology there still remain many research challenges before such systems are deployable in the wearable context (Figure 9 for example). These include form factor reduction, power consumption optimisation and minimal infrastructure implementation. Following on from the proof of concept work presented in this paper a wearable form factor hybrid IEEE 802.5.4-2011 UWB/IMU system has been implemented. The system, illustrated in Figure 10, once fully tested, will be used as a platform for wearable application development.

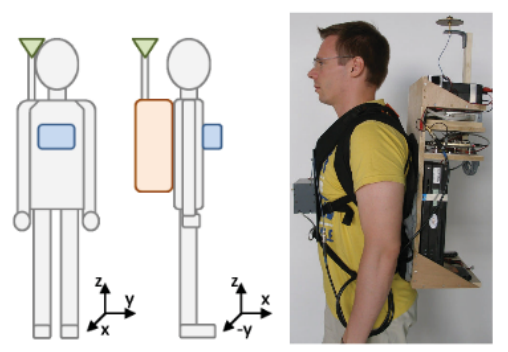

Figure 9. Typical setup for UWB/IMU systems [13]. Photo courtesy of the Karlsruhe Institute of Technology (KIT), Germany, 2013.

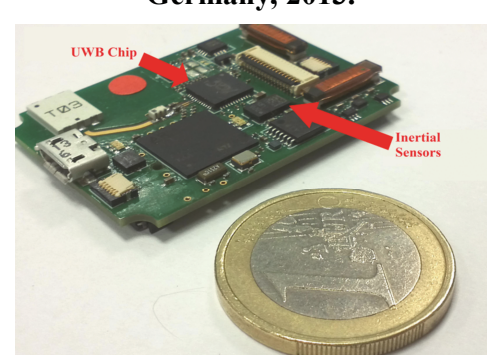

Figure 10. IEEE 802.15.4-2011 UWB/IMU Hybrid Prototype.

\section{REFERENCES}

[1] A. Dey, et al. "Location-based Services," IEEE Pervasive Computing, vol. 9, no. 1, pp. 11-12, March 2010.

[2] L. Kaplan, "Global Node Selection for Localization in a Distributed Sensor Network," IEEE Trans. Aerosp. Electron. Syst., vol. 42, no. 1, pp. 113-135, Jan. 2006.
[3] G. Deak, K. Curran, and J. Condell, "A Survey of Acrive and Passive Indoor Localisation Systems," Computer Communications, vol. 35, no. 16, pp. 1939-1954, Sept. 2012.

[4] Y. Gu, A. Lo, and I. Niemegeers, "A Survey of Indoor Positioning Systems for Wireless Personal Networks," IEEE Communications Surveys \& Tutorials, vol. 11, no. 1, pp. 13$32,2009$.

[5] C. Zhang, et al. "Accurate UWB Indoor Localization System Utilizing Time Difference of Arrival Approach," IEEE Radio Wireless Symp., pp. 515-518, San Diego, USA, Oct. 2006.

[6] M.R. Yuce, T.N. Dissanayake, and H.C. Keong, "Wideband Technology for Medical Detection and Monitoring," from "Recent Advances in Biomedical Engineering", 2009.

[7] Y. Qi, et al., "Using Wearable UWB Radios to Measure Foot Clearance During Walking," IEEE Int. Conf. Eng. Med. Biol. Soc. (EMBC), pp. 5199-5202, Osaka, Japan, July 2013.

[8] A. Fort, et al. "Ultra-Wideband Channel Model for Communication around the Human Body," IEEE J. Sel. Areas Commun., vol. 24, no. 4, pp. 927-933, April 2006.

[9] Q.H. Abbasi, et al. "Radio Channel Characterisation and OFDM-based Ultra Wideband System Modeling for BodyCentric Wireless Networks," IEEE Int. Conf. Body Sensor Networks (BSN), pp. 89-94, Dallas, USA, May 2011.

[10] S. Sczyslo, et al. "Hybrid Localization Using UWB and Inertial Sensors," IEEE Int. Conf. Ultra-Wideband (ICUWB), pp. 89-92, Hannover, Germany, Sept. 2008.

[11] S. Pittet, et al. "UWB and MEMS Based Indoor Navigation," The Journal of Navigation., vol. 61, no. 3, pp. 369-384, July 2008.

[12] J.D. Hol, et al. "Tightly Coupled UWB/IMU Pose Estimation," IEEE Int. Conf. Ultra-Wideband (ICUWB), pp. 688-692, Vancouver, Canada, Sept. 2009.

[13] L. Zwirello, et al., "Sensor Data Fusion in UWB-Supported Inertial Navigation Systems for Indoor Navigation," IEEE Int. Conf. Robotics and Automation (ICRA), pp. 3154-3159, Karlsruhe, Germany, May 2013.

[14] R. Zhang, F. Hoflinger, and L. Reindl, "Inertial Sensor Based Indoor Localization and Monitoring System for Emergency Responders," IEEE Sensors Journal, vol. 13, no. 2, pp. 838-848, Feb. 2013.

[15] M. Walsh, et. al., "A Multi-Technological Approach to Identifying the Reasons for Lateral Drift in Professional and Recreational Darts," IEEE Int. Conf. Body Sensor Networks (BSN), pp. 47-52, Dallas, USA, May 2011.

[16] T. Ya, W. Hongxing, and T. Jingdong, "An Adaptive-Gain Complementary Filter for Real-Time Human Motion Tracking with MARG Sensors in Free-Living Environments," IEEE Trans. Neural Syst. Rehabil. Eng., vol. 21, no. 2, pp. 254-264, March 2013.

[17] M. Gaffney, et al. "An Automated Calibration Tool for High Performance Wireless Inertial Measurement in Professional Sport," IEEE Sensors, pp. 262-265, Limerick, Ireland, Oct. 2011 .

[18] IEEE Working Group 802.15.4a Wireless Medium Access Control (MAC) and Physical Layer (PHY) Specifications for Low-Rate Wireless Personal Area Networks (WPANs). Alternate PHYs. NJ: IEEE, 2007.

[19] T. Ye, et al., "Transceiver-Power Control for 802.15.4a UWB-IR Ranging in the Presence of Multipath Propagation," Int. Conf. Sensor Technol. Applicat. (SENSORCOMM), Barcelona, Spain, Aug. 2013.

[20] M. Gaffney, et al. "A Smart Wireless Inertial Measurement Unit System: Simplifying \& Encouraging Usage of WIMU Technology," Int. Conf. Pervasive Computing Technol. For Healthcare (PervasiveHealth), pp. 198-199, Dublin, Ireland, May 2011 